Y. SHIBUYA

KODAI MATH. J.

1 (1978), 421-439

\title{
REAL SUBMANIFOLDS IN A QUATERNIONIC PROJECTIVE SPACE
}

\author{
By Yhuji SHibuYa
}

\section{§1. Introduction}

The second fundamental form plays a very important role in the study of submanifolds, cf. [1]. From this point of view J. Simons established in [12] a formula for the Laplacian of the length of the second fundamental form, which has enabled us to have a lot of global informations on submanifolds. (See for example [2] and [7].)

On the other hand H. B. Lawson, Jr. introduced in [6] the notion of generalized equators $M_{q, s}^{C}$ and $M_{q, s}^{Q}$ of complex and quaternionic projective spaces, which have stimulated the study of real hypersurfaces of complex and quaternionic projective spaces. (See for example [8] and [11].) His idea, which heavily depends on the theory of fibrations formulated by B. O'Neill in [10], is first to construct a circle bundle over a real hypersurface of the projective space by using the fibrations which are compatible with the Hopf fibrations and second to project known theorems for hypersurfaces of the sphere down to real hypersurfaces of the projective space.

The study of submanifolds of codimension $>1$ of Kählerian and quaternionic Kählerian manifolds is recently started by using Simons' formula. Some of the examples of those submanifolds are invariant submanifolds, totally real (or antiinvariant) submanifolds and totally complex submanifolds. (See for example [3] and [13].) Okumura introduced in [9] the notion of anti-holomorphic submanifolds in studying real submanifolds of codimension $>1$ in a Kählerian manifold using the Hopf fibration.

The purpose of this paper is to characterize a real submanifold $M$ in a quaternionic projective space $Q P^{m}$ by investigating a submanifold $\bar{M}$ in the unit sphere $S^{4 m+3}$, where $\bar{M}=\pi^{-1}(M)$ and the fibration $\pi: \bar{M} \rightarrow M$ is compatible with the Hopf fibration $\tilde{\pi}: S^{4 m+3} \rightarrow Q P^{m}$. In $\S 2$ we prepare fundamental formulas for submanifolds in a quaternionic projective space. In $\S 3$ we review the theory of fibrations, then establish some relations between the connections in the normal bundles of $M$ in $Q P^{m}$ and of $\bar{M}$ in $S^{4 m+3}$. In $\S 4$ we focus our attention to the properties derived from the second fundamental tensors of $M$ and $\bar{M}$. Finally in $\S 5$ we deal with a special class of real submanifolds, called antiquaternionic, in a quaternionic projective space $Q P^{m}$, cf. Definition 5.1, and prove a certain

Recelved August 5, 1977 
pinching problem to determine their character under some conditions.

Manifolds, submanifolds, geometric objects and mappings appearing in this paper are assumed to be differentiable and of class $C^{\infty}$. Indices $A, B$ and $C$ run from 1 to $p . \sum_{A}$ stands for $\sum_{A=1}^{p}$.

\section{§2. Real submanifolds in a quaternionic Kählerian manifold}

Let $\hat{M}$ be a quaternionic Kählerian manifold, cf. [5] with Riemannian metric $\langle$,$\rangle , Riemannian connection D$ and of real dimension $n+p$, which is necessarily a multiple of 4 . Then there exists a 3 -dimensional vector bundle $\Lambda$ of tensors of type $(1,1)$ over $\hat{M}$ satisfying the following conditions (a) and (b):

(a) In any coordinate neighborhood $\hat{O}$ of $\hat{M}$, there is a canonical local basis $\{F, G, H\}$ of $\Lambda$ such that

$$
\begin{array}{lll}
F^{2}=-I, & G^{2}=-I, & H^{2}=-I, \\
G H=-H G=F, & H F=-F H=G, & F G=-G F=H,
\end{array}
$$

where $I$ is the identity tensor field of type $(1,1)$ over $\hat{M}$.

(b) If $\chi$ is a cross-section (local or global) of the bundle $\Lambda$, then $D_{\hat{X}} \chi$ is also a cross-section (local or global respectively) of $\Lambda$, where $\hat{X}$ is an arbitrary vector field in $\hat{M}$. By means of (2.1) the condition (b) is equivalent to the following condition $\left(\mathrm{b}^{\prime}\right)$ :

$\left(\mathrm{b}^{\prime}\right)$ If $\{F, G, H\}$ is a canonical local basis of $\Lambda$, then

$$
\begin{array}{lrl}
D_{\hat{X}} F & =r(\hat{X}) G-q(\hat{X}) H, \\
D_{\hat{X}} G & =-r(\hat{X}) F & +p(\hat{X}) H, \\
D_{\hat{X}} H & =q(\hat{X}) F-p(\hat{X}) G
\end{array}
$$

for any vector field $\hat{X}$ in $\hat{M}$, where $p, q$ and $r$ are certain local 1 -forms.

Let $\hat{O}$ and $\hat{O}^{\prime}$ be arbitrary intersecting coordinate neighborhoods in $\hat{M}$ and let $\{F, G, H\}$ and $\left\{F^{\prime}, G^{\prime}, H^{\prime}\right\}$ be canonical local bases of $\Lambda$ over $\hat{O}$ and $\hat{O}^{\prime}$ respectively. Then $F^{\prime}, G^{\prime}$ and $H^{\prime}$ are linear combinations of $F, G$ and $H$ in $\hat{O} \cap \hat{O}^{\prime}$, that is,

$$
\begin{gathered}
F^{\prime}=s_{11} F+s_{12} G+s_{13} H, \\
G^{\prime}=s_{21} F+s_{22} G+s_{23} H, \quad H^{\prime}=s_{31} F+s_{32} G+s_{33} H
\end{gathered}
$$

with functions $s_{\beta \gamma}(\beta, \gamma=1,2,3)$ in $\hat{O} \cap \hat{O}^{\prime}$. The coefficients $s_{\beta \gamma}$ appearing in (2.3) form an element $s \hat{o} \hat{o}^{\prime}=\left(s_{\beta \gamma}\right)$ of the proper orthogonal group $S O(3)$ of dimension 3 because both $\{F, G, H\}$ and $\left\{F^{\prime}, G^{\prime}, H^{\prime}\right\}$ satisfy (2.1).

Let $M$ be an $n$-dimensional real submanifold with Riemannian metric $g$ immersed isometrically in a quaternionic Kählerian manifold $\hat{M}$ by immersion $i$. 
From now on we use $\imath$ indiscriminately for the differential mapping of the immersion $i$. For any vector fields $X$ and $Y$ in $M$ we have

$$
\langle i X, i Y\rangle=g(X, Y) \text {. }
$$

The tangent space $T_{x}(M)$ to $M$ at $x$ is identified with the subspace of $T_{i(x)}(\hat{M})$. Then $T(M)=\bigcup_{x \in M} T_{x}(M)$ is the tangent bundle to $M$. The normal space $N_{x}(M)$ to $M$ at $x$ is defined as the orthogonal complement to the tangent space $T_{x}(M)$ in $T_{i(x)}(\hat{M})$. Then $N(M)=\bigcup_{x \in M} N_{x}(M)$ is the normal bundle to $M$. If $\hat{M}$ is covered by a system of coordinate neighborhoods $\{\hat{O}\}$, then $M$ is considered to be covered by a system of coordinate neighborhoods $\{O\}$, where $O=\imath^{-1}(\hat{O}) \cap M$. Let $N_{1}, N_{2}$, $\cdots, N_{p}$ form in $O$ an orthonormal local basis of the normal bundle $N(M)$. The transformations of a tangent vector field $X$ and normal vector fields $N_{A}(A=1$, $\cdots, p)$ in $O$ by $F, G$ and $H$ may be written as

$$
\begin{gathered}
F i X=\imath \phi X+\sum_{A} u_{A}(X) N_{A}, \\
G \imath X=\imath \psi X+\sum_{A} v_{A}(X) N_{A}, \quad H i X=i \theta X+\sum_{A} w_{A}(X) N_{A} ; \\
F N_{A}=-i U_{A}+\sum_{B} \phi^{\prime}{ }_{A B} N_{B}, \\
G N_{A}=-i V_{A}+\sum_{B} \phi_{A B}^{\prime} N_{B}, \quad H N_{A}=-i W_{A}+\sum_{B} \theta_{A B}^{\prime} N_{B},
\end{gathered}
$$

where $\phi, \phi$ and $\theta$ are endomorphisms of the tangent bundle to $O, \phi_{A B}^{\prime}, \psi_{A B}^{\prime}$ and $\theta_{A B}^{\prime}$ are endomorphisms of the normal bundle to $O$ and $u_{A}, v_{A}$ and $w_{A}$ are local 1 -forms on $O$ satisfying

$$
u_{A}(X)=g\left(U_{A}, X\right), \quad v_{A}(X)=g\left(V_{A}, X\right), \quad w_{A}(X)=g\left(W_{A}, X\right),
$$

because of (2.4). We easily find that

$$
\phi_{A B}^{\prime}=-\phi_{B A}^{\prime}, \quad \phi_{A B}^{\prime}=-\phi_{B A}^{\prime}, \quad \theta_{A B}^{\prime}=-\theta_{B A}^{\prime} .
$$

Applying $F, G$ and $H$ to (2.5) and making use of (2.1), we obtain the following relations $(2.9) \sim(2.12)$ :

$$
\begin{gathered}
\phi^{2} X=-X+\sum_{A} u_{A}(X) U_{A}, \\
\phi^{2} X=-X+\sum_{A} v_{A}(X) V_{A}, \quad \theta^{2} X=-X+\sum_{A} w_{A}(X) W_{A} ; \\
\phi \theta X=\phi X+\sum_{A} w_{A}(X) V_{A}, \\
\theta \phi X=\phi X+\sum_{A} u_{A}(X) W_{A}, \quad \phi \phi X=\theta X+\sum_{A} v_{A}(X) U_{A}, \\
\theta \phi X=-\phi X+\sum_{A} v_{A}(X) W_{A},
\end{gathered}
$$




$$
\phi \theta X=-\phi X+\sum_{A} w_{A}(X) U_{A}, \quad \phi \phi X=-\theta X+\sum_{A} u_{A}(X) V_{A}
$$

and

$$
u_{A}(\phi X)=-\sum_{B} u_{B}(X) \phi_{B A}^{\prime},
$$

$$
\begin{gathered}
v_{A}(\phi X)=-\sum_{B} v_{B}(X) \psi_{B A}^{\prime}, \quad w_{A}(\theta X)=-\sum_{B} w_{B}(X) \theta_{B A}^{\prime} ; \\
v_{A}(\theta X)=-\sum_{B} w_{B}(X) \psi_{B A}^{\prime}+u_{A}(X),
\end{gathered}
$$

$$
w_{A}(\phi X)=-\sum_{B} u_{B}(X) \theta_{B A}^{\prime}+v_{A}(X), \quad u_{A}(\phi X)=-\sum_{B} v_{B}(X) \phi_{B A}^{\prime}+w_{A}(X),
$$

$$
w_{A}(\psi X)=-\sum_{B} v_{B}(X) \theta_{B A}^{\prime}-u_{A}(X),
$$$$
u_{A}(\theta X)=-\sum_{B} w_{B}(X) \phi_{B A}^{\prime}-v_{A}(X), \quad v_{A}(\phi X)=-\sum_{B} u_{B}(X) \phi_{B A}^{\prime}-w_{A}(X) .
$$

Next applying $F, G$ and $H$ to (2.6) and making use of (2.1), we obtain the following relations $(2.13) \sim(2.16)$ :

$$
\phi U_{A}=-\sum_{B} \phi_{A B}^{\prime} U_{B},
$$

$$
\begin{gathered}
\phi V_{A}=-\sum_{B} \phi_{A B}^{\prime} V_{B}, \quad \theta W_{A}=-\sum_{B} \theta_{A B}^{\prime} W_{B} ; \\
\phi W_{A}=U_{A}-\sum_{B} \theta_{A B}^{\prime} V_{B}, \\
\theta U_{A}=V_{A}-\sum_{B} \phi_{A B}^{\prime} W_{B}, \quad \phi V_{A}=W_{A}-\sum_{B} \psi_{A B}^{\prime} U_{B}, \\
\theta V_{A}=-U_{A}-\sum_{B} \phi_{A B}^{\prime} W_{B}, \\
\phi W_{A}=-V_{A}-\sum_{B} \theta_{A B}^{\prime} U_{B}, \quad \phi U_{A}=-W_{A}-\sum_{B} \phi_{A B}^{\prime} V_{B}
\end{gathered}
$$

and

$$
\begin{gathered}
u_{B}\left(U_{A}\right)=\delta_{A B}+\sum_{C} \phi_{A C}^{\prime} \phi_{C B}^{\prime}, \\
v_{B}\left(V_{A}\right)=\delta_{A B}+\sum_{C} \phi_{A C}^{\prime} \psi_{C B}^{\prime}, \quad w_{B}\left(W_{A}\right)=\delta_{A B}+\sum_{C} \theta_{A C}^{\prime} \theta_{C B}^{\prime} ; \\
v_{B}\left(W_{A}\right)=-\phi_{A B}^{\prime}+\sum_{C} \theta_{A C}^{\prime} \psi_{C B}^{\prime}, \\
w_{B}\left(U_{A}\right)=-\phi_{A B}^{\prime}+\sum_{C} \phi_{A C}^{\prime} \theta_{C B}^{\prime}, \quad u_{B}\left(V_{A}\right)=-\theta_{A B}^{\prime}+\sum_{C} \phi_{A C}^{\prime} \phi_{C B}^{\prime}, \\
w_{B}\left(V_{A}\right)=\phi_{A B}^{\prime}+\sum_{C} \phi_{A C}^{\prime} \theta_{C B}^{\prime}, \\
u_{B}\left(W_{A}\right)=\phi_{A B}^{\prime}+\sum_{C} \theta_{A C}^{\prime} \phi_{C B}^{\prime}, \quad v_{B}\left(U_{A}\right)=\theta_{A B}^{\prime}+\sum_{C} \phi_{A C}^{\prime} \psi_{C B}^{\prime} .
\end{gathered}
$$


Let $\nabla$ denote the Riemannian connection induced in $M$. Then Gauss and Weingarten formulas for the immersion $\imath$ are given respectively by

$$
\begin{gathered}
D_{\imath X} i Y=i \nabla_{X} Y+\sum_{A} g\left(H_{A} X, Y\right) N_{A}, \\
D_{\imath X} N_{A}=-i H_{A} X+D_{X}{ }^{N} N_{A},
\end{gathered}
$$

where $H_{A}$ are the second fundamental tensors associated with the normal vector fields $N_{A}(A=1, \cdots, p)$ and $D^{N}$ denotes the induced connection from $D$ to the normal bundle of $M$ in $\hat{M}$. $D_{X}{ }^{N}$ may be expressed as $D_{X}{ }^{N} N_{A}=\sum_{B} L_{A B}(X) N_{B}$ by the so called third fundamental forms $L_{A B}$. Differentiating (2.5) covariantly and making use of (2.2), we obtain the following relations (2.19) and (2.20):

$$
\begin{aligned}
\left(\nabla_{Y} \phi\right) X= & \sum_{A}\left\{g\left(U_{A}, X\right) H_{A} Y-g\left(H_{A} Y, X\right) U_{A}\right\} \\
& +r(i Y) \phi X-q(i Y) \theta X, \\
\left(\nabla_{Y} \psi\right) X= & \sum_{A}\left\{g\left(V_{A}, X\right) H_{A} Y-g\left(H_{A} Y, X\right) V_{A}\right\} \\
& -r(i Y) \phi X+p(i Y) \theta X, \\
\left(\nabla_{Y} \theta\right) X= & \sum_{A}\left\{g\left(W_{A}, X\right) H_{A} Y-g\left(H_{A} Y, X\right) W_{A}\right\} \\
& +q(i Y) \phi X-p(i Y) \phi X
\end{aligned}
$$

and

$$
\begin{aligned}
\left(\nabla_{Y} u_{A}\right) X= & -g\left(H_{A} Y, X\right)+\sum_{B}\left\{g\left(H_{B} Y, X\right) \phi_{B A}^{\prime}-u_{B}(X) L_{B A}(Y)\right\} \\
& +r(i Y) v_{A}(X)-q(i Y) w_{A}(X), \\
\left(\nabla_{Y} v_{A}\right) X= & -g\left(H_{A} Y, X\right)+\sum_{B}\left\{g\left(H_{B} Y, X\right) \phi_{B A}^{\prime}-v_{B}(X) L_{B A}(Y)\right\} \\
& -r(i Y) u_{A}(X)+p(i Y) w_{A}(X), \\
\left(\nabla_{Y} w_{A}\right) X= & -g\left(H_{A} Y, X\right)+\sum_{B}\left\{g\left(H_{B} Y, X\right) \theta_{B A}^{\prime}-w_{B}(X) L_{B A}(Y)\right\} \\
& +q(i Y) u_{A}(X)-p(i Y) v_{A}(X) .
\end{aligned}
$$

Next differentiating (2.18) covariantly and making use of (2.2), we obtain the following relations (2.21) and (2.22):

$$
\begin{aligned}
& D_{X}{ }^{N} \phi_{A B}^{\prime}=g\left(X, H_{B} U_{A}-H_{A} U_{B}\right)+r(\imath X) \phi_{A B}^{\prime}-q(\imath X) \theta_{A B}^{\prime}, \\
& D_{X}{ }^{N} \phi_{A B}^{\prime}=g\left(X, H_{B} V_{A}-H_{A} V_{B}\right)-r(\imath X) \phi_{A B}^{\prime}+p(\imath X) \theta_{A B}^{\prime}, \\
& D_{X}{ }^{N} \theta_{A B}^{\prime}=g\left(X, H_{B} W_{A}-H_{A} W_{B}\right)+q(\imath X) \phi_{A B}^{\prime}-p(\imath X) \psi_{A B}^{\prime}
\end{aligned}
$$

and 


$$
\begin{aligned}
& \nabla_{X} U_{A}=\phi H_{A} X+\sum_{B}\left\{L_{A B}(X) U_{B}-\phi_{A B}^{\prime} H_{B} X\right\}+r(\imath X) V_{A}-q(i X) W_{A}, \\
& \nabla_{X} V_{A}=\phi H_{A} X+\sum_{B}\left\{L_{A B}(X) V_{B}-\phi_{A B}^{\prime} H_{B} X\right\}-r(\imath X) U_{A}+p(i X) W_{A}, \\
& \nabla_{X} W_{A}=\theta H_{A} X+\sum_{B}\left\{L_{A B}(X) W_{B}-\theta_{A B}^{\prime} H_{B} X\right\}+q(i X) U_{A}-p(\imath X) V_{A} .
\end{aligned}
$$

We now note that (2.20) and (2.22) are equivalent to each other.

Now let the ambient manifold $\hat{M}$ be a quaternionic projective space $Q R^{(n+p) / 4}$ of real dimension $n+p$ equipped with the standard symmetric space metric normalized so that the maximum $Q$-sectional curvature is 4 . Then the curvature tensor $\hat{R}$ of $Q P^{(n+p) / 4}$ is written as

$$
\begin{aligned}
\hat{R}(\hat{X}, \hat{Y}) \hat{Z}= & \langle\hat{Y}, \hat{Z}\rangle \hat{X}-\langle\hat{X}, \hat{Z}\rangle \hat{Y} \\
& +\langle F \hat{Y}, \hat{Z}\rangle F \hat{X}-\langle F \hat{X}, \hat{Z}\rangle F \hat{Y}-2\langle F \hat{X}, \hat{Y}\rangle F \hat{Z} \\
& +\langle G \hat{Y}, \hat{Z}\rangle G \hat{X}-\langle G \hat{X}, \hat{Z}\rangle G \hat{Y}-2\langle G \hat{X}, \hat{Y}\rangle_{G} \hat{Z} \\
& +\langle H \hat{Y}, \hat{Z}\rangle H \hat{X}-\langle H \hat{X}, \hat{Z}\rangle H \hat{Y}-2\langle H \hat{X}, \hat{Y}\rangle H \hat{Z}
\end{aligned}
$$

cf. [5]. Thus, using (2.5) and (2.6), we have the following Codazzi and Ricci equations (2.24) and (2.25) respectively:

$$
\begin{aligned}
\left(\nabla_{X} H_{A}\right) Y & -\left(\nabla_{Y} H_{A}\right) X \\
= & \sum_{B}\left\{L_{A B}(X) H_{B} Y-L_{A B}(Y) H_{B} X\right\} \\
& -g\left(U_{A}, Y\right) \phi X+g\left(U_{A}, X\right) \phi Y-2 g(\phi X, Y) U_{A} \\
& -g\left(V_{A}, Y\right) \phi X+g\left(V_{A}, X\right) \phi Y-2 g(\phi X, Y) V_{A} \\
& -g\left(W_{A}, Y\right) \theta X+g\left(W_{A}, X\right) \theta Y-2 g(\theta X, Y) W_{A}
\end{aligned}
$$

and

$$
\begin{aligned}
& R^{N}(X, Y) N_{A}=\sum_{B}\left\{g\left(\left[H_{A}, H_{B}\right] X, Y\right)\right. \\
& \quad+g\left(U_{A}, Y\right) g\left(U_{B}, X\right)-g\left(U_{A}, X\right) g\left(U_{B}, Y\right)-2 g(\phi X, Y) \phi_{A B}^{\prime} \\
& \quad+g\left(V_{A}, Y\right) g\left(V_{B}, X\right)-g\left(V_{A}, X\right) g\left(V_{B}, Y\right)-2 g(\phi X, Y) \phi_{A B}^{\prime} \\
& \left.\quad+g\left(W_{A}, Y\right) g\left(W_{B}, X\right)-g\left(W_{A}, X\right) g\left(W_{B}, Y\right)-2 g(\theta X, Y) \theta_{A B}^{\prime}\right\} N_{B},
\end{aligned}
$$

where $\left[H_{A}, H_{B}\right]=H_{A} H_{B}-H_{B} H_{A}$ and $R^{N}$ is the curvature tensor of the connection $D^{N}$ in the normal bundle of $M$ in $Q P^{(n+p) / 4}$, i. e.,

$$
R^{N}(X, Y)=D_{X}^{N} D_{Y}^{N}-D_{Y}^{N} D_{X}^{N}-D_{[X, Y]}^{N} .
$$

$R E M A R K$ 2.1. If $M$ is an invariant submanifold of $Q P^{(n+p) / 4}$, 


$$
\begin{aligned}
& R^{N}(X, Y) N_{A} \\
& \quad=\sum_{B}\left\{-2 g(\phi X, Y) \phi_{A B}^{\prime}-2 g(\phi X, Y) \psi_{A B}^{\prime}-2 g(\theta X, Y) \theta_{A B}^{\prime}\right\} N_{B},
\end{aligned}
$$

because $M$ is totally geodesic, cf. [3], and $u_{A}=v_{A}=w_{A}=0(A=1, \cdots, p)$.

\section{$\S 3$. Fibrations and immersions}

Let $S^{n+p+3}(a)$ be the hypersphere of radius $a(>0)$ in the quaternionic space $Q^{(n+p+4) / 4}$ of quaternionic dimension $(n+p+4) / 4$, which is identified with the Euclidean space $R^{n+p+4}$. The unit sphere $S^{m}(1)$ will be simply denoted by $S^{m}$. Let $\tilde{\pi}: S^{n+p+3} \rightarrow Q P^{(n+p) / 4}$ be the natural projection of $S^{n+p+3}$ onto $Q P^{(n+p) / 4}$ defined by the Hopf fibration $S^{3} \rightarrow S^{n+p+3} \rightarrow Q P^{(n+p) / 4}$. As is well known, $S^{n+p+3}$ admits a Sasakian 3 -structure $\{\tilde{\xi}, \tilde{\eta}, \tilde{\zeta}\}$ and each fibre $\tilde{\pi}^{-1}(x)$ of $x \in Q P^{(n+p) / 4}$ is a maximal integral submanifold of the distribution spanned by $\tilde{\xi}$, $\tilde{\eta}$ and $\tilde{\zeta}$, cf. [4]. Therefore, the base space $Q P^{(n+p) / 4}$ admits the induced quaternionic Kählerian structure of constant $Q$-sectional curvature of 4, cf. [5]. We consider a fibration $\pi: \bar{M} \rightarrow M$ which is compatible with the Hopf fibration $\tilde{\pi}: S^{n+p+3} \rightarrow Q P^{(n+p) / 4}$, where $M$ is an $n$-dimensional real submanifold of $Q P^{(n+p) / 4}$ and $\bar{M}=\pi^{-1}(M)$ is a submanifold of $S^{n+p+3}$. More precisely speaking, $\pi: \bar{M} \rightarrow M$ is a fibration with totally geodesic fibres such that the following diagram (3.1) is commutative.

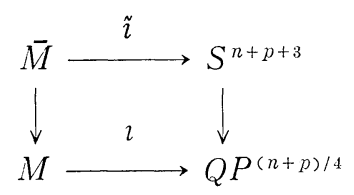

where $\tilde{\imath}: \bar{M} \rightarrow S^{n+p+3}$ and $\imath: M \rightarrow Q P^{(n+p) / 4}$ are isometric immersions.

We are now going to give an example which satisfies the commutative diagram (3.1). Consider the quaternionic space $Q^{(n+5) / 4}$ naturally imbedded in $Q^{(n+p+4) / 4}$ as a totally geodesic submanifold. $Q^{(n+5) / 1}$ is identified with the product space $Q^{q+1} \times Q^{s+1}$, where $q+s=(n-3) / 4$. Hence the product space of two hyperspheres $S^{4 q+3}(a)$ in $Q^{q+1}$ and $S^{4 s+3}(b)$ in $Q^{s+1}$, is naturally considered to be a submanifold in $Q^{(n+5) / 4}$. On the other hand $S^{n+p+3}$ is the unit hypersphere of $Q^{(n+p+4) / 4}$. Thus, when $a^{2}+b^{2}=1$, the product space $M_{4 q+3,4 s+3}=S^{4 q+3}(a) \times S^{4 s+3}(b)$ may be considered to be a submanifold in $S^{n+p+3}$ of codimension $p$. It is now clear that the projected base space $M_{q, s}^{Q}=\pi\left(M_{4 q+3,4 s+3}\right)$ of $M_{4 q+3,4 s+3}$ by the fibration $\pi$, which is compatible with $\tilde{\pi}$, is an example for submanifolds satisfying the commutative diagram (3.1). A submanifold $M$ in $Q P^{(n+p) / 4}$ is sometimes denoted also by $M_{q, s}^{Q}$ if $M$ is congruent to $M_{q, s}^{Q}$ in the sense of quaternionic projective geometry.

Let $\langle$,$\rangle denote indiscriminately the Riemannian metric of S^{n+p+3}$ as well and $\bar{g}$ be the induced Riemannian metric of $\bar{M}$ from $\langle$,$\rangle by the immersion \tilde{i}$. $X^{L}$ denotes the horizontal lift of a vector field $X$ given in the base space for 
each of the fibrations $\tilde{\pi}$ and $\pi$. Since the fibration $\pi$ is compatible with the fibration $\tilde{\pi}$, there exist in $\bar{M}$ unit vector fields $\xi, \eta$ and $\zeta$ which are vertical with respect to the fibration $\pi: \bar{M} \rightarrow M$ and satisfy $\tilde{\imath}(\xi)=\tilde{\xi}, \tilde{\imath}(\eta)=\tilde{\eta}$ and $\tilde{\imath}(\zeta)=\tilde{\zeta}$. By lifing mutually orthonormal local vector fields $N_{A}(A=1, \cdots, p)$ around $x \in M$, we have local normal vector fields $N_{A}{ }^{L}(A=1, \cdots, p)$ to $\bar{M}$ which are also mutually orthonormal around any point of $y \in \bar{M}$ satisfying $\pi(y)=x$. Since the above diagram (3.1) is commutative it is easily verified that $\tilde{\imath}\left(X^{L}\right)=(\imath X)^{L}$.

Let $\bar{D}, \bar{\nabla}, \bar{D}^{N}$ and $\bar{H}_{A}$ denote respectively the Riemannian connection of $S^{n+p+3}$, the induced Riemannian connection of $\bar{M}$ in $S^{n+p+3}$, the induced connection in the normal bundle of $\bar{M}$ in $S^{n+p+3}$ and the second fundamental tensors of $\bar{M}$ in $S^{n+p+3}$ associated with the normal vector fields $N_{A}{ }^{L}(A=1, \cdots, p)$. First we have Gauss and Weingarten formulas for the immersion $\tilde{i}$.

$$
\begin{aligned}
& \bar{D}_{\tau_{\bar{X}} \tilde{\imath}} \bar{Y}=i \nabla_{\bar{X}} \bar{Y}+\sum_{A} \bar{g}\left(\bar{H}_{A} \bar{X}, \bar{Y}\right) N_{A}{ }^{L}, \\
& \bar{D}_{\imath \bar{X}} N_{A}{ }^{L}=-\imath \bar{H}_{A} \bar{X}+\bar{D}_{\bar{X}^{N}} N_{A}{ }^{L} \\
&-\imath \bar{H}_{A} \bar{X}+\sum_{B} \bar{L}_{A B}(\bar{X}) N_{B}{ }^{L},
\end{aligned}
$$

where $\bar{X}, \bar{Y}$ are tangent vectors to $\bar{M}$ and $\bar{L}_{A B}$ are the so called third fundamental forms of the immersion $\tilde{i}$. Next fundamental equations for fibrations $\tilde{\pi}$ and $\pi$ are as follows, cf. [4]:

$$
\begin{aligned}
\bar{D}_{\hat{X}^{L}} \hat{Y}^{L}= & \left(D_{\hat{X}} \hat{Y}\right)^{L} \\
& +\left\langle F^{L} \hat{X}^{L}, \hat{Y}^{L}\right\rangle \tilde{\xi}+\left\langle G^{L} \hat{X}^{L}, \hat{Y}^{L}\right\rangle \tilde{\eta}+\left\langle H^{L} \hat{X}^{L}, \hat{Y}^{L}\right\rangle \tilde{\zeta} \\
\hat{\nabla}_{X} Y^{L}= & \left(\nabla_{X} Y\right)^{L} \\
& +\bar{g}\left(\phi^{L} X^{L}, Y^{L}\right) \xi+\bar{g}\left(\phi^{L} X^{L}, Y^{L}\right) \eta+\bar{g}\left(\theta^{L} X^{L}, Y^{L}\right) \zeta
\end{aligned}
$$

and

$$
\begin{array}{ccc}
\bar{D}_{\widetilde{\xi}} \hat{X}^{L}=-F^{L} \hat{X}^{L}, & \bar{D}_{\widetilde{\eta}} \hat{X}^{L}=-G^{L} \hat{X}^{L}, & \bar{D}_{\widetilde{\zeta}} \hat{X}^{L}=-H^{L} \hat{X}^{L} ; \\
\bar{\nabla}_{\xi} X^{L}=-\phi^{L} X^{L}, & \bar{\nabla}_{r} X^{L}=-\phi^{L} X^{L}, & \bar{\nabla}_{\zeta} X^{L}=-\theta^{L} X^{L} ;
\end{array}
$$

where $X, Y$ are tangent vector fields to $M$ and $\hat{X}, \hat{Y}$ are those to $Q P^{(n+p) / 4}$. If we combine (2.17) with (3.4), (3.5) with (3.2), (2.18) with (3.4) and (3.5) with (3.3), then using (2.5) and (2.6), we have the following relations (3.8) (3.12) because of the commutativity of the diagram (3.1):

$$
\begin{gathered}
\bar{g}\left(\bar{H}_{A} X^{L}, Y^{L}\right)=g\left(H_{A} X, Y\right)^{L} ; \\
\bar{g}\left(\phi^{L} X^{L}, Y^{L}\right)=g(\phi X, Y)^{L}, \\
\bar{g}\left(\phi^{L} X^{L}, Y^{L}\right)=g(\phi X, Y)^{L}, \quad \bar{g}\left(\theta^{L} X^{L}, Y^{L}\right)=g(\theta X, Y)^{L} ; \\
\bar{H}_{A} X^{L}=\left(H_{A} X\right)^{L}-g\left(U_{A}, X\right)^{L} \xi-g\left(V_{A}, X\right)^{L} \eta-g\left(W_{A}, X\right)^{L} \zeta ;
\end{gathered}
$$




$$
\begin{gathered}
\bar{g}\left(\bar{H}_{A} X^{L}, \xi\right)=-g\left(U_{A}, X\right)^{L}, \\
\bar{g}\left(\bar{H}_{A} X^{L}, \eta\right)=-g\left(V_{A}, X\right)^{L}, \quad \bar{g}\left(\bar{H}_{A} X^{L}, \zeta\right)=-g\left(W_{A}, X\right)^{L} ; \\
\bar{L}_{A B}\left(X^{L}\right)=L_{A B}(X)^{L} .
\end{gathered}
$$

Moreover, by (3.6), (3.3) and (2.6), we have

$$
\begin{aligned}
& \left\langle F^{{ }^{L}} N_{A}{ }^{L}, N_{B}{ }^{L}\right\rangle=\phi_{A B}^{\prime}{ }^{L}=-\bar{L}_{A B}(\xi), \\
& \left\langle G^{L} N_{A}{ }^{L}, N_{B}{ }^{L}\right\rangle=\phi_{A B}^{\prime}{ }^{L}=-\bar{L}_{A B}(\eta), \\
& \left\langle H^{L} N_{A}{ }^{L}, N_{B}{ }^{L}\right\rangle=\theta_{A B}^{\prime}{ }^{L}=-\bar{L}_{A B}(\zeta) .
\end{aligned}
$$

On the other hand, since $\{\tilde{\xi}, \tilde{\eta}, \tilde{\zeta}\}$ gives a Sasakian 3 -structure in $S^{n+p+3}, \dot{\xi}, \tilde{\eta}$ and $\tilde{\zeta}$ are mutually orthonormal Killing vector fields. Thus it follows

$$
\bar{D}_{\tilde{\xi}} \tilde{\xi}=\bar{D}_{\tilde{\eta}} \tilde{\eta}=\bar{D}_{\widetilde{\zeta}} \tilde{\zeta}=0
$$

and

$$
\begin{gathered}
\bar{D}_{\tilde{\eta}} \tilde{\zeta}=-\bar{D}_{\widetilde{\zeta}} \tilde{\eta}=\tilde{\xi}, \\
\bar{D}_{\widetilde{\zeta}} \tilde{\xi}=-\bar{D}_{\widetilde{\xi}} \tilde{\zeta}=\tilde{\eta}, \quad \bar{D}_{\widetilde{\xi}} \tilde{\eta}=-\bar{D}_{\tilde{\eta}} \tilde{\xi}=\tilde{\zeta} .
\end{gathered}
$$

Combining (3.14) and (3.15) with (3.2), or using the fact that all fibres of fibrations $\tilde{\pi}$ and $\pi$ are totally geodesic, we have

$$
\begin{aligned}
& \bar{g}\left(\bar{H}_{A} \xi, \xi\right)=\bar{g}\left(\bar{H}_{A} \eta, \eta\right)=\bar{g}\left(\bar{H}_{A} \zeta, \zeta\right)=0, \\
& \bar{g}\left(\bar{H}_{A} \eta, \zeta\right)=\bar{g}\left(\bar{H}_{A} \zeta, \xi\right)=\bar{g}\left(\bar{H}_{A} \xi, \eta\right)=0 .
\end{aligned}
$$

The curvature tensor $\widetilde{R}$ of $S^{n+p+3}$ is given by

$$
\tilde{R}(\tilde{X}, \tilde{Y}) \tilde{Z}=\langle\tilde{Y}, \tilde{Z}\rangle \tilde{X}-\langle\tilde{X}, \tilde{Z}\rangle \tilde{Y},
$$

for any tangent vectors $\tilde{X}, \tilde{Y}$ and $\tilde{Z}$ in $S^{n+p+3}$ because $S^{n+p+3}$ has constant curvature 1 . Thus in this case Ricci equation is reduced to

$$
\bar{R}^{N}(\bar{X}, \bar{Y}) \bar{N}_{A}{ }^{L}=\sum_{B} \bar{g}\left(\left[\bar{H}_{A}, \bar{H}_{B}\right] \bar{X}, \bar{Y}\right) N_{B}{ }^{L},
$$

where $\left[\bar{H}_{A}, \bar{H}_{B}\right]=\bar{H}_{A} \bar{H}_{B}-\bar{H}_{B} \bar{H}_{A}$ and $\bar{R}^{N}$ is the curvature tensor of the connection $\bar{D}^{N}$ in the normal bundle of $\bar{M}$ in $S^{n+p+3}$. By (3.19) we have

LEMMA 3.1. The induced connection $\bar{D}^{N}$ in the normal bundle of $\bar{M}$ in $S^{n+p+3}$ is flat if and only if the second fundamental tensors $\bar{H}_{A}$ and $\bar{H}_{B}$ of $\bar{M}$ commute for any pair $(A, B)(A, B=1, \cdots, p)$.

Now we express the flatness of the connection $\bar{D}^{N}$ in the normal bundle of $\bar{M}$ in terms of conditions imposed on $M$. Using (3.10) and (3.11), we have 


$$
\begin{aligned}
& {\left[\bar{H}_{A}, \bar{H}_{B}\right] X^{L} } \\
&=\left(\left[H_{A}, H_{B}\right] X\right)^{L}+g\left(H_{A} U_{B}-H_{B} U_{A}, X\right)^{L} \xi \\
&+g\left(H_{A} V_{B}-H_{B} V_{A}, X\right)^{L} \eta+g\left(H_{A} W_{B}-H_{B} W_{A}, X\right)^{L} \zeta \\
&-g\left(U_{B}, X\right)^{L} \bar{H}_{A} \xi+g\left(U_{A}, X\right)^{L} \bar{H}_{B} \xi-g\left(V_{B}, X\right)^{L} \bar{H}_{A} \eta \\
&+g\left(V_{A}, X\right)^{L} \bar{H}_{B} \eta-g\left(W_{B}, X\right)^{L} \bar{H}_{A} \zeta+g\left(W_{A}, X\right)^{L} \bar{H}_{B} \zeta .
\end{aligned}
$$

LEMMA 3.2. If the connection $\bar{D}^{N}$ in the normal bundle of $\bar{M}$ in $S^{n+p+3}$ is flat, then the curvature tensor $R^{N}$ of the connection $D^{N}$ in the normal bundle of $M$ in $Q P^{(n+p) / 1}$ is expressed by

$$
\begin{aligned}
& R^{N}(X, Y) N_{A} \\
& \quad=\sum_{B}\left\{-2 g(\phi X, Y) \phi_{A B}^{\prime}-2 g(\phi X, Y) \phi_{A B}^{\prime}-2 g(\theta X, Y) \theta_{A B}^{\prime}\right\} N_{B} .
\end{aligned}
$$

Proof. Taking the inner product of (3.20) and $Y^{L}$ and making use of Lemma 3.1 and (3.11), we have

$$
\begin{aligned}
g\left(\left[H_{A}, H_{B}\right] X, Y\right)= & g\left(U_{A}, X\right) g\left(U_{B}, Y\right)-g\left(U_{B}, X\right) g\left(U_{A}, Y\right) \\
& +g\left(V_{A}, X\right) g\left(V_{B}, Y\right)-g\left(V_{B}, X\right) g\left(V_{A}, Y\right) \\
& +g\left(W_{A}, X\right) g\left(W_{B}, Y\right)-g\left(W_{B}, X\right) g\left(W_{A}, Y\right) .
\end{aligned}
$$

Thus (3.21) follows from (2.25)

Q.E. D.

DEFINITION 3.3. Let $M$ be a real submanifold of a quaternonc Kählerian manifold $\hat{M}$. If $\phi_{A B}^{\prime}, \phi_{A B}^{\prime}$ and $\theta_{A B}^{\prime}$ appearing in (2.6) satisfy the following relation (3.23), we say the structure induced in the normal bundle of $M$ in $\hat{M}$ is parallel.

$$
\begin{array}{ll}
D_{X}{ }^{N} \phi_{A B}^{\prime}= & r(\imath X) \phi_{A B}^{\prime}-q(\imath X) \theta_{A B}^{\prime}, \\
D_{X}{ }^{N} \phi_{A B}^{\prime}=-r(\imath X) \phi_{A B}^{\prime} & +p(\imath X) \theta_{A B}^{\prime}, \\
D_{X}{ }^{N} \theta_{A B}^{\prime}=q(\imath X) \phi_{A B}^{\prime}-p(\imath X) \phi_{A B}^{\prime} . &
\end{array}
$$

LEMMA 3.4. Let $M$ be a real submanıfold of a quaternonic Kählerian manfold $\hat{M}$. The structure induced in the normal bundle of $M$ in $\hat{M}$ is parallel if and only if the following relations in (3.24) are valid:

$$
H_{B} U_{A}=H_{A} U_{B}, \quad H_{B} V_{A}=H_{A} V_{B}, \quad H_{B} W_{A}=H_{A} W_{B},
$$

for all $A, B=1, \cdots, p$.

Proof. This is straightforward by (2.21)

Q.E. D.

LEMMA 3.5. If the connection $\bar{D}^{N}$ in the normal bundle of $\bar{M}$ in $S^{n+p+3}$ is flat, then the structure induced in the normal bundle of $M$ in $Q P^{(n+p) / 4}$ is parallel. 
Proof. Taking the inner product of (3.20) and $\xi$ and making use of Lemma 3.1, (3.16) and (3.17), we get $H_{B} U_{A}=H_{A} U_{B}$. The same process derives the rest of the relations in (3.24). Lemma 3.4 leads to the conclusion. Q. E. D.

THEOREM 3.5. In order that the connection $\bar{D}^{N}$ in the normal bundle of $\bar{M}$ in $S^{n+p+3}$ is flat, it is necessary and sufficient that the curvature tensor $R^{N}$ of the connection $D^{N}$ in the normal bundle of $M$ on $Q P^{(n+p) / 4}$ is expressed by (3.21), that is,

$$
\begin{aligned}
& R^{N}(X, Y) N_{A} \\
& \quad=\sum_{B}\left\{-2 g(\phi X, Y) \phi_{A B}^{\prime}-2 g(\phi X, Y) \phi_{A B}^{\prime}-2 g(\theta X, Y) \theta_{A B}^{\prime}\right\} N_{B}
\end{aligned}
$$

and the structure induced in the normal bundle of $M$ in $Q P^{(n+p) / 4}$ is parallel.

Proof. Necessity follows from Lemmas 3.2 and 3.5. If we prove $\left[\bar{H}_{A}, \bar{H}_{B}\right]$ $=0$, then by Lemma 3.1 we see the condition is sufficient. Since (3.21) holds, we have $\bar{g}\left(\left[\bar{H}_{A}, \bar{H}_{B}\right] X^{L}, Y^{L}\right)=0$ by (3.20), (3.8) and (3.11). On the other hand, since (3.23) or, equivalently, (3.24) holds, we have

$$
\bar{g}\left(\left[\bar{H}_{A}, \bar{H}_{B}\right] X^{L}, \xi\right)=\bar{g}\left(\left[\bar{H}_{A}, \bar{H}_{B}\right] X^{L}, \eta\right)=g\left(\left[\bar{H}_{A}, \bar{H}_{B}\right] X^{L}, \zeta\right)=0
$$

by (3.20), (3.16) and (3.17). Finally $\bar{H}_{A} \xi, \bar{H}_{A} \eta$ and $\bar{H}_{A} \zeta$ are horizontal because of (3.16) and (3.17), therefore so are $\bar{H}_{A} \bar{H}_{B} \xi, \bar{H}_{A} \bar{H}_{B} \eta$ and $\bar{H}_{A} \bar{H}_{B} \zeta$. Thus

$$
\begin{aligned}
& \bar{g}\left(\left[\bar{H}_{A}, \bar{H}_{B}\right] \xi, \xi\right)=\bar{g}\left(\left[\bar{H}_{A}, \bar{H}_{B}\right] \eta, \eta\right)=\bar{g}\left(\left[\bar{H}_{A}, \bar{H}_{B}\right] \zeta, \zeta\right) \\
= & \bar{g}\left(\left[\bar{H}_{A}, \bar{H}_{B}\right] \eta, \zeta\right)=\bar{g}\left(\left[\bar{H}_{A}, \bar{H}_{B}\right] \zeta, \xi\right)=g\left(\left[\bar{H}_{A}, \bar{H}_{B}\right] \xi, \eta\right)=0 .
\end{aligned}
$$

Since $\bar{H}_{A}$ are symmetric tensors, we may conclude $\left[\bar{H}_{A}, \bar{H}_{B}\right]=0$. Q. E. D.

\section{$\S 4$. The second fundamental tensors $H_{A}$ of $M$ and $\bar{H}_{A}$ of $\bar{M}$.}

First we investigate the relations between the mean curvature vector flelds $\mu$ of $M$ and $\bar{\mu}$ of $\bar{M}$. The mean curvature vector field $\mu$ of $M$ is defined by

$$
\mu=\frac{1}{n} \sum_{A}\left(\operatorname{trace} H_{A}\right) N_{A}
$$

and $\bar{\mu}$ of $\bar{M}$ by

$$
\bar{\mu}=\frac{1}{n+3} \sum_{A}\left(\operatorname{trace} \bar{H}_{A}\right) N_{A}{ }^{L} .
$$

It is well known that $\mu$ and $\bar{\mu}$ are independent of the choices of the local bases $\left\{N_{A}\right\}$ and $\left\{N_{A}{ }^{L}\right\}$.

LEMMA 4.1. At any point of $\bar{y} \in \bar{M}$ we have

$$
\left(\operatorname{trace} \bar{H}_{A}\right)(\bar{y})=\left(\operatorname{trace} H_{A}\right)^{L}(\bar{y})=\left(\operatorname{trace} H_{A}\right) \pi(\bar{y}) \text {. }
$$


Consequently $M$ is minimal in $Q P^{(n+p) / 4}$ if and only if $\bar{M}$ is minimal in $S^{n+p+3}$.

Proof. Let $\left\{E_{1}, \cdots, E_{n}\right\}$ be an orthonormal basis of $T_{\pi(\bar{y})}(M)$. We choose an orthonormal basis $\left\{\bar{E}_{1}, \cdots, \bar{E}_{n+3}\right\}$ of $T_{\bar{y}}(\bar{M})$ in such a way that $\bar{E}_{i}=E_{\imath}{ }^{2}$ for $\imath=1, \cdots, n, \bar{E}_{n+1}=\xi, \bar{E}_{n+2}=\eta$ and $\bar{E}_{n+3}=\zeta$. Then because of (3.8) and (3.16) we get

$$
\begin{aligned}
\operatorname{trace} \bar{H}_{A} & =\sum_{\imath=1}^{n+3} \bar{g}\left(\bar{H}_{A} \bar{E}_{\imath}, \bar{E}_{\imath}\right) \\
& =\sum_{\imath=1}^{n} \bar{g}\left(\bar{H}_{A} E_{\imath}{ }^{L}, E_{\imath}{ }^{L}\right)+\bar{g}\left(\bar{H}_{A} \xi, \xi\right)+\bar{g}\left(\bar{H}_{A} \eta, \eta\right)+\bar{g}\left(\bar{H}_{A} \zeta, \zeta\right) \\
& =\sum_{\imath=1}^{n} g\left(H_{A} E_{\imath}, E_{\imath}\right)^{L}=\left(\operatorname{trace} H_{A}\right)^{L}
\end{aligned}
$$

Thus the first equality in (4.3) is proved. The second equality in (4.3) follows from the definition of lifted functions in a fibred Riemannian space, cf. [4].

By Lemma 4.1, we have

Q.E.D.

COROLLALY 4.2. The following relation (4.4) holds between the mean curvature vector fields $\mu$ of $M$ and $\bar{\mu}$ of $M$,

$$
\bar{\mu}=\frac{n}{n+3} \mu^{L}
$$

Proof. This is straightforward by (4.1) and (4.2).

Q.E.D.

LEMMA 4.3. If the mean curvature vector field $\bar{\mu}$ of $\bar{M}$ is parallel with respect to the induced connectioo $\bar{D}^{N}$ in the normal bundle of $\bar{M}$ in $S^{n+p+3}$, then the mean curvature vector field $\mu$ of $M$ is parallel with respect to the induced connection $D^{N}$ in the normal bundle of $M$ in $Q P^{(n+p) / 4}$.

Proof. Since (3.12) is valid, we have

$$
\begin{aligned}
(n+3) \bar{D}_{X}{ }^{N} \bar{\mu} & =\sum_{A}\left\{\left(X^{L} \text { trace } \bar{H}_{A}\right) N_{A}{ }^{L}+\left(\operatorname{trace} \bar{H}_{A}\right) \bar{D}_{X}{ }^{{ }^{N}} N_{A}{ }^{L}\right\} \\
& =\sum_{A}\left\{X^{L}\left(\operatorname{trace} H_{A}\right)^{L} N_{A}{ }^{L}+\left(\operatorname{trace} H_{A}\right)^{L}\left(D_{X}{ }^{N} N_{A}\right)^{L}\right\} \\
& =\sum_{A}\left\{\left(X \operatorname{trace} H_{A}\right) N_{A}+\left(\operatorname{trace} H_{A}\right) D_{X}{ }^{N} N_{A}\right\}^{L} \\
& =n\left(D_{X}{ }^{N} \mu\right)^{L} .
\end{aligned}
$$

Thus $\bar{D}_{X^{L}}{ }^{N} \bar{\mu}=0$ implies $D_{X}{ }^{N} \mu=0$.

Q. E. D.

We shall now relate the lengths of the second fundamental tensors $H_{A}$ of $M$ and $\bar{H}_{A}$ of $\bar{M}$. Choose orthonormal bases $\left\{E_{i}\right\}$ and $\left\{\bar{E}_{i}\right\}$ in the same way as in the proof of Lemma 4.1. Using (3.10), (3.11) and (3.17), we see that 
$\operatorname{trace} \bar{H}_{A}^{2}=\sum_{\imath=1}^{n+3} \bar{g}\left(\bar{H}_{A}^{2} \bar{E}_{\imath}, \bar{E}_{\imath}\right)$

$$
\begin{aligned}
= & \sum_{\imath=1}^{n} \bar{g}\left(\bar{H}_{A}\left(\left(H_{A} E_{\imath}\right)^{L}-g\left(U_{A}, E_{\imath}\right)^{L} \xi-g\left(V_{A}, E_{\imath}\right)^{L} \eta-g\left(W_{A}, E_{\imath}\right)^{L} \zeta\right), E_{\imath}{ }^{L}\right) \\
& +\bar{g}\left(\bar{H}_{A} \xi, \bar{H}_{A} \xi\right)+\bar{g}\left(\bar{H}_{A} \eta, \bar{H}_{A} \eta\right)+\bar{g}\left(\bar{H}_{A} \zeta, \bar{H}_{A} \zeta\right) \\
= & \sum_{\imath=1}^{n}\left\{\bar{g}\left(\bar{H}_{A}\left(H_{A} E_{\imath}\right)^{L}, E_{\imath}{ }^{L}\right)+g\left(U_{A}, E_{\imath}\right)^{L^{2}}+g\left(V_{A}, E_{\imath}\right)^{L^{2}}+g\left(W_{A}, E_{\imath}\right)^{L^{2}}\right\} \\
& +\bar{g}\left(\bar{H}_{A} \xi, \bar{H}_{A} \xi\right)+\bar{g}\left(\bar{H}_{A} \eta, \bar{H}_{A} \eta\right)+\bar{g}\left(\bar{H}_{A} \zeta, \bar{H}_{A} \zeta\right) \\
= & \sum_{\imath=1}^{n}\left\{g\left(H_{A}{ }^{2} E_{\imath}, E_{\imath}\right)^{L}+g\left(U_{A}, E_{\imath}\right)^{L^{2}}+g\left(V_{A}, E_{\imath}\right)^{L^{2}}+g\left(W_{A}, E_{\imath}\right)^{\left.L^{2}\right\}}\right. \\
& +g\left(U_{A}, U_{A}\right)^{L}+g\left(V_{A}, V_{A}\right)^{L}+g\left(W_{A}, W_{A}\right)^{L} \\
= & \left\{\operatorname{trace} H_{A}{ }^{2}+2 g\left(U_{A}, U_{A}\right)+2 g\left(V_{A}, V_{A}\right)+2 g\left(W_{A}, W_{A}\right)\right\}^{L},
\end{aligned}
$$

Hence we have the following relation (4.7):

$$
\begin{aligned}
& \sum_{A} \operatorname{trace} \bar{H}_{A}{ }^{2} \\
& \quad=\left(\sum_{A} \operatorname{trace} H_{A}{ }^{2}\right)^{L}+2 \sum_{A}\left\{g\left(U_{A}, U_{A}\right)+g\left(V_{A}, V_{A}\right)+g\left(W_{A}, W_{A}\right)\right\}^{L} .
\end{aligned}
$$

Next we rewrite (4.7) using the scalar curvatures $r$ of $M$ and $\bar{r}$ of $\bar{M}$. Since the curvature tensor $\hat{R}$ of $Q P^{(n+p) / 4}$ is expressed by (2.23) Gauss equation for the immersion $\imath: M \rightarrow Q P^{(n+p) / 4}$ is given by

$$
\begin{aligned}
g(R(X, Y) Z, W)=g(Y, Z) g(X, W)-g(X, Z) g(Y, W) \\
\quad+g(\phi Y, Z) g(\phi X, W)-g(\phi X, Z) g(\phi Y, W)-2 g(\phi X, Y) g(\phi Z, Y) \\
\quad+g(\phi Y, Z) g(\phi X, W)-g(\phi X, Z) g(\phi Y, W)-2 g(\phi X, Y) g(\phi Z, Y) \\
\quad+g(\theta Y, Z) g(\theta X, W)-g(\theta X, Z) g(\theta Y, W)-2 g(\theta X, Y) g(\theta Z, Y) \\
\quad+\sum_{A}\left\{g\left(H_{A} Y, Z\right) g\left(H_{A} X, W\right)-g\left(H_{A} X, Z\right) g\left(H_{A} Y, W\right)\right\}
\end{aligned}
$$

where $R$ denotes the curvature tensor of $M$. Thus using (2.5) and (2.9), we have

$$
\begin{aligned}
r= & n(n+8)-3 \sum_{A}\left\{g\left(U_{A}, U_{A}\right)+g\left(V_{A}, V_{A}\right)+g\left(W_{A}, W_{A}\right)\right\} \\
& +\sum_{A}\left(\operatorname{trace} H_{A}\right)^{2}-\sum_{A} \operatorname{trace} H_{A}{ }^{2} .
\end{aligned}
$$

If $M$ is minimal, substituting (4.9) with trace $H_{A}=0$ into (4.7), it follows

$$
\begin{aligned}
& \sum_{A} \operatorname{trace} \bar{H}_{A}{ }^{2} \\
& \quad=n(n+8)-r-\sum_{A}\left\{g\left(U_{A}, U_{A}\right)+g\left(V_{A}, V_{A}\right)+g\left(W_{A}, W_{A}\right)\right\} .
\end{aligned}
$$


On the other hand because of (3.18), Gauss equation for the immersion $\tilde{\imath}: \bar{M} \rightarrow$ $S^{n+p+3}$ is given by

$$
\begin{aligned}
& \bar{g}(\bar{R}(\bar{X}, \bar{Y}) \bar{Z}, \bar{W})=\bar{g}(\bar{Y}, \bar{Z}) \bar{g}(\bar{X}, \bar{W})-\bar{g}(\bar{X}, \bar{Z}) \bar{g}(\bar{Y}, \bar{W}) \\
& \quad+\sum_{A}\left\{\bar{g}\left(\bar{H}_{A} \bar{Y}, \bar{Z}\right) \bar{g}\left(\bar{H}_{A} \bar{X}, \bar{W}\right)-\bar{g}\left(\bar{H}_{A} \bar{X}, \bar{Z}\right) \bar{g}\left(\bar{H}_{A} \bar{Y}, \bar{W}\right)\right\},
\end{aligned}
$$

where $\bar{R}$ denotes the curvature tensor of $\bar{M}$. Thus we have

$$
\bar{r}=(n+2)(n+3)+\sum_{A}\left(\operatorname{trace} \bar{H}_{A}\right)^{2}-\sum_{A} \operatorname{trace} \bar{H}_{A}{ }^{2} .
$$

Substituting (4.9) and (4.12) into (4.7) and making use of Lemma 4.1, we obtain the following relation (4.13):

$$
\bar{r}+(3 n-6)=r^{L}+\sum_{A}\left\{g\left(U_{A}, U_{A}\right)+g\left(V_{A}, V_{A}\right)+g\left(W_{A}, W_{A}\right)\right\}^{L} .
$$

LEMMA 4.4. Inequalities

$$
\sum_{A} \operatorname{trace} \bar{H}_{A}^{2} \geqq\left(\sum_{A} \operatorname{trace} H_{A}{ }^{2}\right)^{L}
$$

and

$$
\bar{r}+(3 n-6) \geqq r^{L}
$$

are always valid. In both cases equalities hold if and only if the submanifold $M$ of $Q P^{(n+p) / 4}$ is invariant, that $2 s, M$ is $Q P^{n / 4}$.

If $\tilde{\imath}$ is a totally geodesic immersion, the relation (4.7) leads to

LEMMA 4.5. Suppose $\tilde{i}$ is a totally geodesic ımmersion of a Riemannian manifold $\bar{M}$ into $S^{n+p+3}$. Then $\imath$ is also a totally geodesic immersion of $M$ into $Q P^{(n+p) / 4}$ and $M$ is an invariant submanifold of $Q P^{(n+p) / 4}$, that $\imath$, $M$ is $Q P^{n / 4}$.

Proposition 4.6. In a quaternionic projective space $Q P^{(n+p) / 4}$, a compact totally geodesıc submanifold $M$ of real codimension $p<(n+9) / 12$ or equivalently of the scalar curvature satısfying $r>n(n+8)-(6 p+n) /(2-1 / p)$ is necessarily an invariant submanifold and consequently a quaternıonc projective space $Q P^{n / 4}$.

Proof. From (2.15) we have

$$
\begin{aligned}
& \sum_{A} g\left(U_{A}, U_{A}\right)=p+\sum_{C} \phi_{A C}^{\prime} \phi_{C A}^{\prime} \leqq p, \\
& \sum_{A} g\left(V_{A}, V_{A}\right)=p+\sum_{C} \phi_{A C}^{\prime} \psi_{C A}^{\prime} \leqq p, \\
& \sum_{A} g\left(W_{A}, W_{A}\right)=p+\sum_{C} \theta_{A C}^{\prime} \theta_{C A}^{\prime} \leqq p .
\end{aligned}
$$

Since $M$ is totally geodesic, it follows from (4.7)

$$
\begin{aligned}
\sum_{A} \operatorname{trace} \bar{H}_{A}^{2} & =2 \sum_{A}\left\{g\left(U_{A}, U_{A}\right)+g\left(V_{A}, V_{A}\right)+g\left(W_{A}, W_{A}\right)\right\}^{L} \\
& \leqq 6 p<(n+3) /(2-1 / p),
\end{aligned}
$$


and from (4.10)

$$
\sum_{A} \operatorname{trace} \bar{H}_{A}^{2} \leqq n(n+8)-r-3 p<(n+3) /(2-1 / p)
$$

Considering Lemma 4.1, we see that $\bar{M}$ is minimal. Thus applying Simons' result, cf. [12], to $\bar{M}$, we obtain that $\bar{M}$ is totally geodesic. By Lemma $4.5 M$ is necessarily $Q P^{n / 1}$.

Q.E. D.

COROLlaRy 4.7. If $n \neq 0(\bmod 4)$, there is no $n$-dimensional compact totally geodesic submanifold of codimension $p<(n+9) / 12$ or of the scalar curvature $r$ $>n(n+8)-(6 p+n) /(2-1 / p)$ in a quatermonc projective space $Q P^{(n+p) / 4}$.

THEOREM 4.8. If a compact minumal submanifold $M$ of real codimension $p$ of a quaternonic projective space $Q P^{(n+p) / 4}$ satisfies either of the following equivalent mequalities

$$
\begin{gathered}
\sum_{A} \operatorname{trace} H_{A}{ }^{2}<(n-12 p+9) /(2-1 / p), \\
r>n(n+8)-(6 p+n) /(2-1 / p),
\end{gathered}
$$

then $M$ is a quaternomic projective space $Q P^{n / 4}$.

Proof. From (4.7) and (4.14) it follows

$$
\begin{aligned}
\sum_{A} \operatorname{trace} \bar{H}_{A}{ }^{2} \leqq & \sum_{A}\left(\operatorname{trace} H_{A}{ }^{2}\right)^{L}+6 p \\
& <(n-12 p+9) /(2-1 / p)+6 p=(n+3) /(2-1 / p)
\end{aligned}
$$

and from (4.10)

$$
\begin{aligned}
\sum_{A} \operatorname{trace} H_{A}^{2} \leqq n(n+8)-r-3 p \\
<(n+3) /(2-1 / p)
\end{aligned}
$$

Since $M$ is minimal so is $\bar{M}$ by Lemma 4.1. Thus in both cases applying Simons' result to $\bar{M}$, we see that $\bar{M}$ is totally geodesic. By Lemma $4.5 M$ is necessarily $Q P^{n / 4}$.

Q.E.D.

\section{§5. Anti-quaternionic submanifolds.}

If $M$ is an invariant submanifold of a quaternionic Kählerian manifold $\hat{M}$, then at any point $x \in M$ we have

$$
F T_{x}(M) \perp N_{x}(M), \quad G T_{x}(M) \perp N_{x}(M), \quad H T_{x}(M) \perp N_{x}(M),
$$

or equivalently

$$
F N_{x}(M) \perp T_{x}(M), \quad G N_{x}(M) \perp T_{x}(M), \quad H N_{x}(M) \perp T_{x}(M) .
$$

On the other hand if $M$ is a totally real (or anti-invariant) submanifold of a quaternionic Kählerian manifold $\hat{M}$, then at any point $x \in M$ we have 


$$
F T_{x}(M) \perp T_{x}(M), \quad G T_{x}(M) \perp T_{x}(M), \quad H T_{x}(M) \perp T_{x}(M) .
$$

In this section we shall consider a submanifold $M$ which satisfies the following conditions at any point $x \in M$ :

$$
F N_{x}(M) \perp N_{x}(M), \quad G N_{x}(M) \perp N_{x}(M), \quad H N_{x}(M) \perp N_{x}(M) .
$$

DeFinition 5.1. Let $M$ be a submamfold of a quaternomic Kählerian manfold $\hat{M}$ with canonical local basıs $\{F, G, H\}$. We call $M$ anti-quaternonic submanifold of $\hat{M}$ if $M$ satisfies the condition (5.4) at any point $x \in M$.

REMARK 5.2. In other words, $M$ is an anti-quaternionic submanifold of $\hat{M}$ if and only if all of $\phi_{A B}^{\prime}, \phi_{A B}^{\prime}$ and $\theta_{A B}^{\prime}$ in (2.6) vanish.

LEMma 5.3. A submanifold $M$ of a quaternonic Kählerian manfold $\hat{M}$ cannot be totally real and anti-quatermonic at the same time.

Proof. Let $M$ and $\hat{M}$ be of real dimensions $n$ and $n+p$ respectively. Suppose $M$ is totally real and anti-quaternionic at the same time, it easily follows from (5.3) and (5.4) that $p$ should satisfy both $p \geqq 3 n$ and $n \geqq 3 p$. This is a contradiction.

Q.E.D.

REMARK 5.4. In the complex case the corresponding condition to anti-quaternionic is anti-holomorphic. It is possible that a submanifold of a Kählerian manifold is totally real and antiholomorphic at the same time. For example consider the real axis in the complex plane.

Rroposition 5:5. Let $M$ be an n-dimensional ant-quatermonc submanfold of a quaternomic projective space $Q P^{(n+p) / 4}$ of real dimension $n+p$ and let $\pi$ : $\bar{M} \rightarrow M$ be a fibration which is compatible with the Hopf fibration $\tilde{\pi}: S^{n+p+3} \rightarrow$ $Q P^{(n+p) / 1}$. Then the mean curvature vector field $\mu$ of $M$ is parallel with respect to the induced connection $D^{N}$ in the normal bundle of $M i n Q P^{(n+p) / 4}$ if and only if the mean curvature vector field $\bar{\mu}$ of $\bar{M}$ is parallel with respect to the induced connection $\bar{D}^{N}$ in the normal bundle of $\bar{M}$ in $S^{n+p+3}$.

Proof. By Lemma 4.3 we have only to prove that $\bar{\mu}$ is parallel if $\mu$ is parallel. From (4.2) it follows that

$$
\begin{aligned}
& \bar{D}_{\tilde{\xi}^{N}} \bar{\mu}=\sum_{A}\left\{\left(\xi \operatorname{trace} \bar{H}_{A}\right) N_{A}{ }^{L}+\left(\operatorname{trace} \bar{H}_{A}\right) \bar{D}_{\tilde{\xi}^{N}} N_{A}{ }^{L}\right\}, \\
& \bar{D}_{\gamma^{N}}{ }^{N}=\sum_{A}\left\{\left(\eta \operatorname{trace} \bar{H}_{A}\right) N_{A}{ }^{L}+\left(\operatorname{trace} \bar{H}_{A}\right) \bar{D}_{\eta}{ }^{N} N_{A}{ }^{L}\right\}, \\
& \bar{D}_{\zeta}^{N} \bar{\mu}=\sum_{A}\left\{\left(\zeta \operatorname{trace} \bar{H}_{A}\right) N_{A}{ }^{L}+\left(\operatorname{trace} \bar{H}_{A}\right) \bar{D}_{\zeta}{ }^{N} N_{A}{ }^{L}\right\} .
\end{aligned}
$$

Because of Lemma 4.1 trace $\bar{H}_{A}$ are invariant functions with respect to $\xi$, $\eta$ and $\zeta$, that is

$$
\xi \operatorname{trace} \bar{H}_{A}=\eta \operatorname{trace} \bar{H}_{A}=\zeta \operatorname{trace} \bar{H}_{A}=0
$$


Moreover since $M$ is anti-quaternionic, by (3.13) and Remark 5.2 we have

$$
\begin{aligned}
& \bar{D}_{\xi^{N}}^{N} N_{A}{ }^{L}=\sum_{B} \bar{L}_{A B}(\xi) N_{B}{ }^{L}=-\sum_{B}\left(\phi_{A B}^{\prime}\right)^{L} N_{B}{ }^{L}=0, \\
& \bar{D}_{r_{r}}^{N} N_{A}{ }^{L}=\sum_{B} \bar{L}_{A B}(\eta) N_{B}{ }^{L}=-\sum_{B}\left(\phi_{A B}^{\prime}\right)^{L} N_{B}{ }^{L}=0, \\
& \bar{D}_{\zeta}^{N} N_{A}{ }^{L}=\sum_{B} \bar{L}_{A B}(\zeta) N_{B}{ }^{L}=-\sum_{B}\left(\theta_{A B}^{\prime}\right)^{L} N_{B}{ }^{L}=0 .
\end{aligned}
$$

Thus we obtain

$$
\bar{D}_{\xi^{N}} \bar{\mu}=\bar{D}_{\eta}^{N} \bar{\mu}=\bar{D}_{\zeta}^{N} \bar{\mu}=0 .
$$

Considering (4.5) and (5.8), we see that $\bar{\mu}$ is parallel with respect to the connection $\bar{D}^{N}$.

Q.E.D.

Proposition 5.6. If $M$ is an antz-quatermomc submamfold of $Q P^{(n+p) / 4}$, we have (3.24), namely

$$
H_{B} U_{A}=H_{A} U_{B}, \quad H_{B} V_{A}=H_{A} V_{B}, \quad H_{B} W_{A}=H_{A} W_{B} .
$$

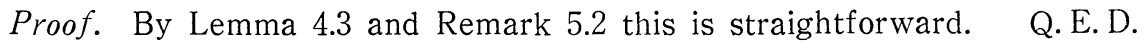

Proposition 5.7. Let $M$ be an antz-quatermonc submanifold of $Q P^{(n+p) / 4}$. The connection $D^{N}$ in the normal bundle of $M$ in $Q P^{(n+p) / 4}$ us flat if and only if the connection $\bar{D}^{N}$ in the normal bundle of $\bar{M}$ in $S^{n+p+3}$ is flat. 3.5 .

Proof. This follows easily from Lemma 3.2, Proposition 5.6 and Theorem

THEOREM 5.8. Let $M$ be an n-dimensional compact minimal antiquaternonic submanifold of a quaternionc projective space $Q P^{(n+p) / 4}$ of real dimension $n+p$. If either of the following equivalent inequalities

$$
\begin{gathered}
\sum_{A} \operatorname{trace} H_{A}{ }^{2} \leqq(n-12 p+9) /(2-1 / p) \\
r \geqq n(n+8)-(6 p+n) /(2-1 / p)
\end{gathered}
$$

holds everywhere on $M$, then $M$ is $M_{q, s}^{Q} \imath n Q P^{(n+1) / 4}$ for some $q$ and s satisfynng $q+s=(n-3) / 4$.

If $p=1$, Theorem 5.8 is Lawson's result as stated in the following Corollary 5.9 because a hypersurface in $Q P^{(n+1) / 4}$ is anti-quaternionic. (See Theorem in [6])

COROLlARY 5.9. Let $M$ be an n-dimensional compact minimal hypersurface of $Q P^{(n+1) / 4}$. If either of the equivalent inequalities

$$
\text { trace } H^{2} \leqq n-3
$$

$$
r \geqq n^{2}+7 n-6
$$


holds everywhere on $M$, then $M$ is $M_{q, s}^{Q}$ for some $q$ and s satısfying $q+s=(n-3) / 4$.

Proof of Theorem 5.8. Since $M$ is anti-quaternionic, by (4.14) we have

$$
\sum_{A} g\left(U_{A}, U_{A}\right)=\sum_{A} g\left(V_{A}, V_{A}\right)=\sum_{A} g\left(W_{A}, W_{A}\right)=p .
$$

Thus from (4.7) and (5.9) it follows

$$
\begin{aligned}
\sum_{A} \operatorname{trace} \bar{H}_{A}{ }^{2} & =\sum_{A}\left(\operatorname{trace} H_{A}{ }^{2}\right)^{L}+6 p \\
& \leqq(n+3) /(2-1 / p)
\end{aligned}
$$

and from (4.10) and (5.10)

$$
\begin{aligned}
\sum_{A} \operatorname{trace} \bar{H}_{A}^{2} & =n(n+8)-r-3 p \\
& \leqq(n+3) /(2-1 / p) .
\end{aligned}
$$

Since $M$ is minimal, $\bar{M}$ is also minimal by Lemma 4.1. If in (5.14) and (5.15) equalities are not satisfied, we see that $M$ is $Q P^{n / 4}$ by Theorem 4.8. But this is impossible because $M$ is anti-quaternionic. Thus equalities necessarily hold in both (5.14) and (5.15). From the result of Chern-do Carmo-Kobayashi, cf [2], it follows that $\bar{M}$ is isometric to $M_{m, n+3-m}=S^{m}(\sqrt{m /(n+3)}) \times S^{n+3-m}(\sqrt{(n+3-m) /(n+3)})$ in $S^{n+3+1}$ and the second fundamental tensor $\bar{H}$ of $M_{m, n+3-m}$ in $S^{n+3+1}$ is parallel. Considering the fibration $\pi$ is compatible with that of $\tilde{\pi}$, we see the second fundamental tensor $H$ of $M$ in $Q P^{(n+1) / 4}$ and each of $\phi, \phi$ and $\theta$ appearing in (2.5) are commutative. Thus $m=4 q+3$ and $n+3-m=4 s+3$ for some $q$ and $s$ satisfying $q+s=(n-3) / 4$. (For detail see [11]) Hence $M=\pi\left(M_{4 q+3,4 s+3}\right)=M_{q, s}^{Q}$.

Q.E.D.

\section{BIBLIOGRAPHY}

[1] B. -Y. Chen, Geometry of Submanfolds, Marcel Dekker Inc., New York, 1973.

[2] S. S. Chern, M. do Carmo and S. Kobayashi, Minimal submanifolds with second fundamental form of constant length, Functional analysis and related fields, Springer, (1970), 59-75.

[3] S. Funabashi, Totally complex submanifolds of a quaternınıc Kählerıan manifold, to appear.

[4] S. IshinARA AND M. Konishi, Differential geometry of fibred spaces, Study Group of Differential Geometry, Tokyo, 1973.

[5] S. IshinARA, Quaternionıc Kählerıan manifolds, J. Differentıal Geometry, 9 (1974), 483-500.

[6] H.B. Lawson, Jr., Rigidity theorems in rank 1 symmetric spaces, J. Differential Geometry, 4 (1970), 349-357.

[7] K. Nomizu And B. Smyth, A formula of Simons' type and Hypersurfaces with constant mean curvature, J. Differential Geometry, 3 (1969), 367-377.

[8] M. OKumura, On some real hypersurfaces of a complex projective space, Transactions of the Amer. Math. Soc., 212 (1975), 355-364. 
-9] M. OKumura, Submanifolds of real codimension $p$ of a complex projective space, Atti della Accademia Nazionale dei Lincei, 58 (1975), 544-555.

[10] B. O'NeILl, The fundamental equations of a submersion, Michigan Math. J., 13 (1966), 459-469.

[11] J. S. PAK, Real hypersurfaces in quaternionıc Kählerian manıfolds with constant Q-sectional curvature, Tokyo Institute of Technology, 1976.

[12] J. Simons, Minimal varieties in riemannian manifolds, Ann. of Math., 88 (1968), $62-105$.

[13] K. Yano And M. Kon, Anti-invariant Submanifolds, Marcel Dekker Inc,. New York, 1976.

Tokyo Institute of Technology 
